\title{
Anomaly Detection and Attribution in Network
}

\author{
K Viswak Raj, M Mukesh, J.Kalaivani
}

\begin{abstract}
In this article, we address the problem of not only id entifying phenomena, but also attributing the phenomenon to the movement that induces it. This causes to a combinatorial optimisation problem, which is prohibitively expensive. Instead we design two anomaly detection algorithms that are small in complexity. The first is based on the system for cross-entropy (CE), which identifies flow anomalies and labels flow anomalies. The second algorithm detects anomalies through GLRT on aggregated flow transformation a compact low-dimensional representation of raw traffic flows. The two algorithms complement each other and allow the network operator to use the algorithm for flow aggregation first so that device irregularities can be identified easily. After discovery of an exception, the user Can analyse further that individual flows are anomalous using CE-based algorithm. We perform extensive performance tests and trials on synthetic and semi-synthetic data with our algorithms, as well as real Internet traffic data gathered from the MAWI database, and finally make recommendations as to their usability.

Keywords: cross-entropy (CE), MAWI.
\end{abstract}

\section{INTRODUCTION}

ANOMALIES from the' standard' in INTRODUCTION Data The network's predicted behaviour is characteristics of that network. Deviate the behaviours can include irregular practicesnetwork scaning of weak ports / networks, threats T CP SYN flooding, DDoS amplification attacks, etc. spurious network failure traffic. The identification of phenomena has many uses in various research fields. These include identification of shifts in sensor networks, detection of IoT networks location spoofing, tracking of fraud etc. In this research, we focus on anomalies throughout data networks, but the concept and algorithms that we are creating can also be extended to other domains There are various works which have tried to solve the network anomaly detection problem. Many functions of granularities ranging Information found from packets to flows to sessions in past works (see for a recent analysis). Similarly, with identification of irregularities in a subset of flows, several models were used to analyse these features. We can also assign in other words; can we determine what anomalous subset of flows is? Such important aspects were not concerned with using a single coding according to our best knowledge beforehand. The method so far has been to evaluate flow by flow, a rece nt example of this.

Revised Manuscript Received on April 02, 2020.

* Correspondence Author

K Viswak Raj*, Dept of Computer Science and Engineering, SRM Institute of Science and Technology Chennai. India vishwakrajk@gmail.com

M Mukesh, Dept of Computer Science and Engineering, SRM Institute of Science and Technology Chennai. India mukeshmohan162@gmail.com

Dr.J.Kalaivani, Dept of Computer Science and Engineering, SRM Institute of Science and Technology Chennai. India kalaivaj@srmist.edu.in

(c) The Authors. Published by Blue Eyes Intelligence Engineering and Sciences Publication (BEIESP). This is an open access article under the CC BY-NC-ND license (http://creativecommons.org/licenses/by-nc-nd/4.0/)
This method is obviously suboptimal, as the combined number of measurements of flows does not decompose into individual flows; As such, the joint density should be considered by a rational statistical analysis and the detection algorithms built based on this number. This type of problem of mutual anomaly identification and attribution can be interpreted as a combinatorial research theory, which can only be understood Unless the number of flows is low, the number of theories to be evaluated is exponential in the number of flows. When it exists hypothesis of algorithm start work on this. This greatlySimplifies the complexity of the problem, as we don't have to compare all possible model sets. The construction of an optimal detector for our model also requires exponential complexity, a question that we tackle by developing two diff erent ways to perform the detection tasks.The first algorithm solves the problem of optimisation through an updated Cross-Entropy version Provide stability to properly maintain and manage the tradeoff between exploration and discovery. The second algorith $\mathrm{m}$, hereafter related to as the Flow aggregation algorithm, we first implement a transformation in traffic flow resulting in a compact low-dimensional representation; This helps us to build a rapid anomaly detection algorithm with a linear flow complexity. Although the transformation maintains the ability to detect a nomalies, it does lead to some lack of individual flow infor mation.

\section{EXISTING SYSTEMS}

The transition of traditional energy networks into smart grids will further revolutionize the efficiency, performance, and manageability of the energy industry. Nonetheless, there are serious security vulnerabilities to enhanced synchronization of power grid infrastructure for bidirectional communications

Disadvantages:

- Detects only if, when the transaction is handled, an unauthorized person injects data into shared data.

\section{III.}

\section{PROPOSED SYSTEM}

The Proposed System is used not only to track the anomalous phenomena, but also to assign the phenomenon to the flows that triggered it. This leads to a prohibitively expensive combinatorial optimization problem, we made two anomaly detection algorithms

with a low complexity. The first is based on a crossentropy ( CE) system, which identifies flow anomalies and attributes.

\section{Advantages:}

- After observation of a phenomenon, the operator may further examine which individual flows are anomalous.

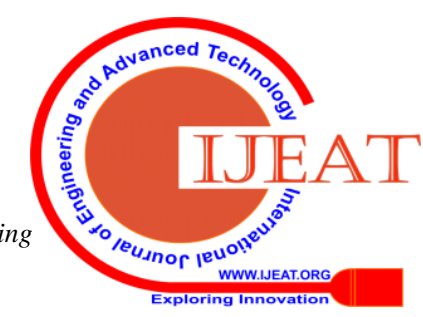


IV.

\section{PROBLEM STATEMENT}

In transiently connected rush hour gridlock correspondence systems, we built up another measurable structure for irregularity recognition, by means of Markov Chain demonstrating the traffic highlights being checked. We have defined the ideal problem of recognition of irregularities by createing two ideals Calculations to discovery. Cross Entropy's primary calculation not only identifies the presence of an oddity, but also credits it to the sub-set of streams is odd. The resulting estimation is based on a stream conglomeration that assumes a traditional, low-dimensional representation of the sources of coarse flow. We assessed the result, the carrying out of our measurements by way.

\section{MODULE DESCRIPTION}

\section{- $\quad$ Register}

Save Your Account. A check register is the document used to record all receipts, cash payments and cash outlays over a period in accounting.

\section{- Login}

A login is a set of credentials used to get a user authenticated. Which consist most often of a username and a password. Nevertheless, other details may include a password, such as a PIN number, passcode, or passphrase. Many logins require a biometric identification, including fingerprint scans or retina scans.

\section{Upload and Send File}

For their records in the cloud, pass a database to the server and move the data from one hub to the next site. Give the record to Destination.

\section{- $\quad$ View Traffic}

Network traffic alludes to the amount of knowledge that passes over a network for a given time purpose. For the most part, system knowledge is expressed in system packets, which send the heap into the system. Service traffic is the basic section for service flow prediction, traffic control and leisure arrangements.

\section{- View anomaly detection}

Anomaly detection is an important task of analysing information that detects unusual or abnormal information from a given dataset. Unit execute oddity detection (NBAD) is the constant testing of an exclusive unit for unusual instances or patterns. NBAD is a vital piece of system conduct investigation (NBA) that provides an additional layer of security for that given by the usual enemy

\section{VI.}

\section{RESULT}

The detection output is quantified through the ROC curves, which depict the probability of detection against tThe probability of false alarm for different threshold settings $\gamma$, such that,

$$
\begin{aligned}
& \text { - Probability of detection }:=\operatorname{Pr}\left(\Lambda\left(\mathbf{S}_{1: T}^{(1: K)}\right) \geq \gamma \mid \mathcal{H}_{1}\right) \text {, } \\
& \text { - Probability of false alarm }:=\operatorname{Pr}\left(\Lambda\left(\mathbf{S}_{1: T}^{(1: K)}\right) \geq \gamma \mid \mathcal{H}_{0}\right)
\end{aligned}
$$

\section{VII.}

\section{CONCLUSION}

We also developed a new component in this work mathematical method for the identification of anomalies throughout temporally linked traffic communication networks, by Markov Chain analysis of the traffic features being tracked. We formulated the optimal problem in detection among ano malies as the Nyman Pearson Probably test and developed two optimal detection algorithms. Cross Entropy-based first algorithm not only detects

\section{ACKNOWLEGEMENT}

The author wishes to thank SRM Science and Technology Institute for their assistance.

\section{REFERENCES}

1. J. Y. Koh, I. Nevat, D. Leong, and W.-C. Wong, "Geo-spatial location spoofing detection for Internet of Things," IEEE Internet Things J., vol. 3, no. 6, pp. 971-978, Dec. 2016.

2. F. Iglesias and T. Zseby, "Analysis of network traffic features for anomaly detection," Mach. Learn., vol. 101, nos. 1-3, pp. 59-84, 2014.

3. J. Wang and I. C. Paschalidis, "Statistical traffic anomaly detection in time-varying communication networks," IEEE Trans. Control Netw. Syst., vol. 2, no. 2, pp. 100-111, Jun. 2015.

\section{AUTHOR PROFILE}

K Viswak Raj, Dept of Computer Science and Engineering, SRM Institute of Science and Technology chennai India vishwakrajk@gmail.com.

M Mukesh, Dept of Computer Science and Engineering, SRM Institute of Science and Technology chennai India mukeshmohan162@gmail.com.

Dr.J.Kalaivani, Department of Computer Science and Engineering, Kattankulathur Campus, SRM Institute of Science and Technology chennai India

kalaivani.j@ktr.srmuniv.ac.in

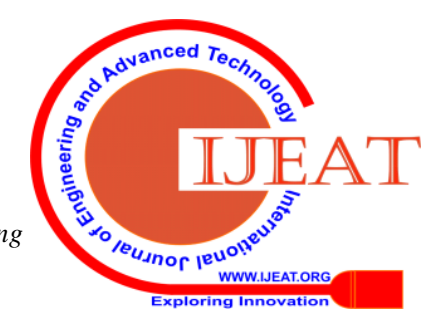

